\title{
"ESCRÚPULOS RELIGIOSOS EXTREMOS" Y DELIRIO MÍSTICO - RELIGIOSO: ENTRE LA DEVOCIÓN CRISTIANA Y LA PSICOPATOLOGÍA EN COLOMBIA, 1920-1960
}

\author{
Jairo Gutiérrez Avendaño \\ Universidad Católica Luis Amigó, Colombia. \\ Email: jairo.gutierrezav@amigo.edu.co \\ ORCID iD: https://orcid.org/0000-0002-0642-0722
}

Recibido: 2 abril 2018; Aceptado: 5 julio 2019.

Cómo citar este artículo/Citation: Gutiérrez Avendaño, Jairo (2020), “'Escrúpulos religiosos extremos’ y delirio místico - religioso: entre la devoción cristiana y la psicopatología en Colombia, 1920-1960", Asclepio, 72(1): p298. https://doi.org/10.3989/asclepio.2020.07.

RESUMEN: Objetivo: comprender la semiología del delirio místico-religioso y la incidencia del contenido sociocultural en la configuración de los síntomas en la psicopatología moderna en Colombia, 1920-1960. Metodología: investigación histórico-hermenéutica, mediante análisis de una serie de historias clínicas del antiguo Manicomio Departamental de Antioquia y del Asilo Psiquiátrico San Isidro del Valle del Cauca. Resultados: entre las psicopatologías más recurrentes en las que se manifestaron los delirios se encuentra el grupo de las manías, principalmente la intermitente, psicosis maniaco-depresiva y melancolía; seguido por la esquizofrenia paranoide, y también fue frecuente en la psicosis puerperal o posparto; asimismo, se presentó en la parálisis general progresiva (neurosífilis) y en el síndrome cerebral agudo o estado de confusión agudo, nosografía moderna dada al delirio. Según las historias clínicas, la exaltación de ideas religiosas confluye en un delirio polimorfo. Los síntomas más recurrentes fueron las alucinaciones visuales y auditivas, logorrea o locuacidad, insomnio, negativismo alimentario acompañado de miedo a ser envenenado, excitación psicomotora o hiperquinética, accesos de furia controlados con camisa de fuerza y otros medios de contención, intento de suicidio y tendencia a la fuga de la casa, por lo tanto, se consideraba de peligrosidad para sí mismos y para los demás.

PALABRAS CLAVE: delirio místico-religioso; devoción cristiana; alucinaciones; éxtasis; delirio polimorfo.

\section{“EXTREME RELIGIOUS SCRUPLES” OR MYSTICAL DELIRIUM - RELIGIOUS: BETWEEN CHRISTIAN DEVO- TION AND PSYCHOPATHOLOGY IN COLOMBIA, 1920-1960}

\begin{abstract}
Objective: To understand the semiotics of mystical-religious delirium and the incidence of sociocultural content in the configuration of symptoms in modern psychiatry in Colombia, 1920-1960. Methodology: hermeneutic historical, research by analysis of a series of case histories of the old Madhouse Department of Antioquia and the Psychiatric Asylum San Isidro del Valle del Cauca. Results: Among the most frequent psychopathologies in which delusions demonstrated is the group of hobbies, mainly intermittent, manic depression and melancholy; followed by paranoid schizophrenia, and was also common in puerperal or postpartum psychosis; He also appeared in general paresis (neurosyphilis) and acute brain syndrome or acute confusional state, delirium given modern nosography. According to medical records, the exaltation of religious ideas comes together in a polymorph delirium. The most frequent symptoms were visual and auditory hallucinations, logorrea or talkativeness, insomnia, accompanied food negativism fear of being poisoned, psychomotor or hyperkinetic excitement, fits of rage controlled straitjacket and other means of containment, attempted suicide and trend the escape of the house, therefore it was considered dangerous to themselves and others.
\end{abstract}

KEY WORDS: mystical-religious delirium; Christian devotion; hallucinations; ecstasy; polymorph delirium.

Copyright: ( 2020 CSIC. Este es un artículo de acceso abierto distribuido bajo los términos de la licencia de uso y distribución Creative Commons Reconocimiento 4.0 Internacional (CC BY 4.0). 


\section{ESCRÚPULO RELIGIOSO, ÉXTASIS MÍSTICO E HISTERIA}

El "escrúpulo" (diminutivo scrupus, piedrecilla en el zapato), según el influjo de la teología moral, es la "inquietud de ánimo provocada por la duda acerca de si algo es bueno o malo, correcto o incorrecto, verdadero o falso" (Genicot, 1931/1992, p. 46). De ahí que, no proviene únicamente del ámbito del primer alienismo, porque está presente en épocas anteriores; por ejemplo, Huertas lo rastrea desde el siglo IV en Agustín de Hipona, quien "propició, en buena medida, el origen de una presencia duradera e incuestionable de las obsesiones en el marco cultural de la iglesia católica latina" (Huertas, 2014, p. 1399). Así, se destaca el origen de la diferenciación entre "posesión" (possessio = captura del mal o pérdida de autocontrol) y "obsesión" (obssessio = asedio o temor al mal). De este modo, la presencia de escrúpulos en la conciencia moral aparece en la literatura como un síntoma fundamental en la historia del concepto de obsesión.

Prosiguió una larga tradición de esta doctrina y, en el siglo XVII, Taylor, capellán de la corona inglesa, lo definió en su Ductor Dubitantium, or the Rule of Conscience... (1660), como "un gran problema de la mente que proviene de un pequeño motivo y una gran indisposición, por el cual la conciencia, aunque suficientemente determinada por los argumentos apropiados, no se atreve a actuar, o si lo hace, no puede descansar" (p. 9).

En el siglo XVIII, el teólogo Duguet publicó su Traité des scrupules (1718), en el que afirma que "es una duda en materia moral que no tiene fundamento o que es muy a la ligera... Depende de la persuasión que llena la conciencia de problemas y de perplejidad" (Duguet, 1718, p. 3).

En L'Encyclopédie (1751), compilada por Diderot y D'Alembert, en su entrada de "Scrupule" aportada por De Jaucourt, define que "este vicio es el resultado de la falta de inteligencia, la poca sensatez, la pusilanimidad, la ignorancia y una falsa opinión de la religión y de Dios" (De Jaucourt, 1751, p. 815).

En el siglo XIX, Guislain, pionero de la psiquiatría belga, en sus Leçons orales sur les phrénopathies (1852), según la clasificación de Esquirol, en la categoría del "delirio acusador", incluye el escrúpulo melancólico; así como en la del delirio religioso, en la que distingue entre la demonolatría (posesión y culto) y la demonofobia (temor continuo al infierno con excesivos escrúpulos religiosos) (Guislain, 1852, p. 130).

También se propuso una terapéutica teológica, como la de Grimes, en su Traité des scrupules (1854), en el que propone remedios como las oraciones contra los escrúpulos; la confesión; amor y corrección fraternales; consejos sobre el motivo de sus acciones; evitar pensar en la predestinación; prevenir las degeneraciones y las tentaciones (Grimes, 1854, pp. 62-86).

Según el jesuita Genicot, en su Institutiones theologiae moralis (1896), la conciencia escrupulosa se define, siguiendo a Alfonso de Ligoro, como aquella "que, por motivos leves, sin causa o fundamento razonable, a menudo teme el pecado donde de hecho no existe" (Genicot, 1931 / 1992, p. 46). Estas ideas fijas, así como el miedo de haber cometido pecado mortal, guardan relación con los contenidos de los delirios de condenación y autoinculpación, como se encuentra en casos de asilos psiquiátricos de Colombia en la primera mitad del siglo XX.

En el campo de la psicología, Delacroix en sus Etudes d'histoire et de psychologie du mysticisme (1908), basado en testimonios autobiográficos, cartas y otros documentos de consagrados místicos cristianos, como son Teresa de Ávila, Madame Guyon, Francisco de Sales y Juan de la Cruz, se propuso diferenciar la experiencia mística, asumida como capacidad excepcional, forma de vida de grandes creadores y simplificadores del mundo; más allá de los estados patológicos que usualmente le han atribuido. Así lo critica en tanto que "los bajos místicos, degenerados sin genio, neurópatas sin poder intelectual y voluntario, alienados conocidos por un séquito de piadosos, pululan y son legión..." (Delacroix, 1908, p. 3). En efecto, expuso que en el campo de la medicina se consideraba que el éxtasis místico era un "accidente histérico, la inmovilidad cataléptica, la obliteración sensorial, la obnubilación mental, las visiones que aparecen al principio o al final, la frecuente amnesia consecutiva, parecen asegurar este diagnóstico" (Delacroix, 1908, pp. 338-339).

Para una autoridad en este campo, como Tanquerey, en su Précis de théologie ascétique et mystique (1930), el éxtasis místico se caracteriza por tres fases:

a) El desfallecimiento que se produce dulcemente, y causa en el alma una herida dolorosa y deli- 
ciosa a la vez; el Esposo hace sentir al alma su presencia, mas solo por poco tiempo; el alma querría gozar de esa presencia de continuo, y padece por la privación... b) El arrobamiento se apodera del alma con impetuosidad y violencia, tanto que no se puede resistir a él... c) El vuelo del espíritu, el cual es tan impetuoso que parece separar al alma del cuerpo, y que no se puede resistir" (Tanquerey 1930, pp. 979-980).

Por otra parte, según estudios sobre la obsesión y psicastenia (debilidad del alma), nosología acuñada por el neurólogo Pierre Janet en 1893, el éxtasis oscilaba entre el estado de ánimo escrupuloso y el estado mental histérico (Janet, 1903, p. 23). La psicastenia se caracterizaba por periodos de miedo o ansiedad, obsesiones, ideas fijas, tics, sensaciones de insuficiencia, autoacusación y sensaciones extrañas de rareza, irrealidad y despersonalización.

Como se mencionó, con respecto de los escrúpulos religiosos, estos síntomas concuerdan con los diagnósticos de delirio místico-religioso en Colombia. Por ejemplo, en una revista médica se publicó la traducción de un artículo de la revista Journal des Practiciens (1907), sobre una crítica a la atribución histérica de los místicos, en el que se cuestionaba el reduccionismo de estos seres, en tanto que "histérico o degenerado no hay término medio. Desde que exista tendencia al misticismo hay tara mental: tal es la fórmula" (Varieté, 1907, pp. 177-179); de este modo, es establecía una distinción entre el éxtasis y la histeria, porque se consideraba que esta última no era una enfermedad, sino "un estado particular del dinamismo nervioso; en ella hay exceso de energía; solo que esta no se emplea útilmente y se convierte en convulsiones, contracturas, incoherencias en la conducta" (Varieté, 1907, pp. 177-179).

\section{MEDIDAS ASILARES}

Pinel, en su Traité Médico-Philosophique sur I'aliénation mentale (1809), en la Sección IV, "Policía interior y reglas a seguir en los establecimientos dedicados a los alienados", estableció "Precauciones que se deben tomar ante la exaltación extrema de las opiniones religiosas", sobre las cuales indicaba que debían considerarse bajo estricto criterio médico y desechar cualquier interpretación popular y política, asimismo que se investigara si tenía incidencia impedir la exaltación de ideas y sentimientos de dicha índole en la curación de los "escrúpulos religiosos extremos" propios de la "melancolía devocional" (Pinel, 1809 / 2012, p. 508). Estas fijaciones eran un riesgo porque muy a menudo conducían a la desesperación y al suicidio en alienadas del hospicio de La Salpêtrière, donde Pinel oficiaba como médico director.

Una segunda precaución era oponerse a los desdenes y el engreimiento de la arrogancia que, según Pinel, eran actitudes propias de la manía devocional, para lo cual se usaba un tono autoritario, con el fin de dominar su carácter, táctica que funcionaba en algunos casos, porque "muchos otros resisten con una energía inflexible a todos los medios de represión, bajo pretexto de que es mejor desobedecer a los hombres que al Ser supremo, del cual piensan recibir inspiraciones inmediatas" (Pinel, 1809 / 2012, p. 509).

Una tercera medida debía tomarse para que las "melancólicas devotas" no presenciaran rituales religiosos, en particular en la enfermería, porque podrían repetirse situaciones que ocasionaron recaídas por el estremecimiento frente al dolor, la enfermedad y la muerte, así como el recuerdo de la pérdida de un ser querido.

Finalmente, Pinel cuestionaba si ¿̇e debía consentir los pedidos reiterados que hacen las melancólicas por devoción de conservar, cerca de ellas, libros religiosos, para poder leerlos asiduamente y buscar nuevos consuelos de su confesor habitual? Esto se resolvía como lo indicaba la experiencia clínica, la cual mostraba que este tipo de contenidos eran un riesgo por ser "el medio más seguro para perpetuar la alienación, o incluso para volverla incurable, y cuanto uno más consiente esas lecturas, menos llega a calmar las inquietudes y los escrúpulos" (Pinel, $1809 / 2012$, p. 510), como ocurrió con una alienada en calma desde hace algún tiempo, que volvió a alterarse cuando "un libro que le prestaron le recordó que cada persona tenía su ángel guardián: a la siguiente noche se creyó rodeada por un coro de ángeles, se adujo haber escuchado una música celestial y haber tenido revelaciones. Le sacamos su libro, que fue quemado, pero no dejó de tener una segunda recaída y el tratamiento se volvió más largo y más equívoco" (Pinel, 1809/2012, p. 511). En cuanto a la visita de sacerdotes al hospicio de alienadas, los casos expuestos por Pinel mostraban que las confesiones generaban nuevas inquietudes en las alienadas y en otros casos se resistían porque, como en el caso an- 
terior, solo obedecían a Dios, a Jesucristo o a otra divinidad.

Si bien en Colombia se mantuvo un alienismo tardío basado en el tratamiento manicomial francés, la administración de los asilos por parte de comunidades religiosas, emulaba su forma de administrar los claustros monacales y conventuales, por lo tanto, existió un fuerte influjo del dogma cristiano en la concepción de la locura, de su expiación de la culpa y la resignación a este designio impuesto por la divinidad. De hecho, la devoción se consideraba un síntoma de recuperación y la blasfemia de perturbación (Gutiérrez, 2019, p. 155).

Por cierto, en la definición del asilo psiquiátrico como "institución total", Goffman muestra que el orden interior, influido por la comunidad religiosa, concordaba con La regla benedictina, para el estricto funcionamiento de los monasterios, acogida por la mayoría de los fundados durante la Edad Media, desde el siglo VI (Goffman, 2009, pp. 31-35).

\section{DELIRIO MÍSTICO - RELIGIOSO Y MANÍA DEVOCIONAL EN COLOMBIA}

La teoría del delirio y la monomanía de Esquirol, fue apropiada por Nicolás Buendía en su estudio clínico y médico-legal de 1893, en el que atribuía a causas ocasionales dicho trastorno: "los excesos de todo género, la lectura de ciertos libros, las desgracias domésticas, las profundas preocupaciones del espíritu, la exaltación religiosa, en una palabra, todo lo que puede impresionar profundamente las facultades intelectuales puede dar nacimiento a la enfermedad" (Buendía, 1893, p. 10).

Asimismo, la tesis de doctorado en medicina de Francisco Alvarado, analizó casos remitidos a la Oficina Médico Legal de Cundinamarca en 1904, en los que se manifestó el "delirio de persecución" y definió que se trataba de una "forma de enajenación mental caracterizada por un delirio parcial y sistematizado en el cual ciertas ideas llevan el sello de la insania, mientras que en otros puntos la razón queda aparentemente intacta" (Alvarado, 1904, 12). Basado en Esquirol, estableció que el delirio tenía cuatro periodos: 1. De interpretación delirante, 2. De alucinaciones sensoriales y desórdenes de la sensibilidad general, 3. De ideas de grandeza y transformación de la personalidad, 4. De demencia.
Se observa la confluencia del delirio de persecución con el místico, el de grandeza, el erótico y el de celos, a los que Alvarado dedica un apartado de su tesis a cada uno y consideró que la influencia de las ideas religiosas exageradas en la producción de la locura era innegable. El alienado antiguamente atribuía las voces y alucinaciones a presencias demonológicas y a creencias de condenación e impenitencia; pero, según él, en los tiempos modernos "acusa á las corporaciones, á los cuerpos colectivos como los jesuitas, los fracmasones, etc., ó bien da á sus acusaciones explicaciones científicas por medio de la física, la química, la electricidad o el magnetismo". Asimismo, acude a Esquirol para afirmar que "Ias ideas amorosas son fijas, dominantes, como son fijas y dominantes las ideas religiosas en la teomanía ó en la lipemanía religiosa" (Alvarado, 1904, p. 25).

En el Asilo de Indigentes y Locos de San Diego, en el departamento de Cundinamarca entre 1907 y 1919, era frecuente el diagnóstico de Manía/Monomanía/Melancolía mística-religiosa, en cincuenta casos de más de mil registros en los libros de entradas y salidas. Asimismo, en el Asilo de Locas de Bogotá entre 1918 y 1919, hubo 7 casos de Manía religiosa en más de 300 registros.

En el Manicomio departamental de Antioquia, entre 1945 y 1960, se encontraron 18 casos entre manía religiosa y delirio místico, de una muestra de más de 900 registros. En el Hospital Psiquiátrico San Isidro del Valle del Cauca, en los primeros diez años de funcionamiento, 1958 - 1968, aparecen 7 casos de delirio místico, de una serie de 152 registros, pero no como enfermedad sino como síntoma principalmente asociado a la esquizofrenia. Estas cifras, aunque sean poco representativas, interesan para mostrar la pervivencia de experiencias extáticas, alucinatorias, histéricas, de culpa y penitencia, durante la primera mitad del siglo XX en Colombia.

\section{PATOLOGIZACIÓN DEL FANATISMO}

En 1837 se registró uno de los primeros peritazgos psiquiátricos realizados por una junta médica en Medellín, Colombia, en el cual se diagnosticó manía intermitente padecida por un sacerdote controversial de la época, estuvo basado en antecedentes hereditarios, en causas morales como los sustos de tedio por la vida, las dificultades domésticas y la devoción exagerada (De los Ríos, 1981, p. 170). 
Este episodio, también fue comentado por Rosselli, en cuanto al imaginario de la psicopatología en sus inicios, la cual "depende en gran parte de la actitud hacia la vida que adopta la población en general, incluyendo muchos médicos. Se consideran los desórdenes mentales como resultado del pecado moral y la conducta contra la ética: creen que la pérdida de la razón es un justo castigo que la cólera divina descarga sobre el individuo" (Rosselli, 1968, p. 506). No en vano, la moderación de la devoción se consideró un síntoma de mejoría o el rechazo de la misma, perturbación mental. De hecho, en algunos municipios los sacerdotes rendían declaración de la "conducta moral y religiosa" del enfermo.

El fanatismo religioso fue considerado entre las causas psíquicas de la locura, por parte de médicos como Putnam quien, en 1913, afirmaba que la raza colombiana estaba influida por atavismos ancestrales cargados de "supersticiones, los amuletos, la adivinación y la brujería con sus agüeros y la creación fantástica de un espíritu maligno que persigue a las criaturas humanas...", los cuales degeneraban en locura (Putnam, 1913, pp. 248-259). En estas mismas atribuciones sobre el carácter fanático, reivindicador y sectario del pueblo colombiano, coincidieron autores adeptos a la teoría de la degeneración, como Jiménez (1920, p. 27), Uribe (1923, pp. 188-198) y Vasco (1948, p. 83).

Según las narrativas clínicas, se observa que en el delirio místico-religioso, la mayoría de casos se presentaron en mujeres dedicadas a los oficios domésticos, en muchas se prestó atención a su "apariencia personal" como descripción relacionada con los síntomas, en la que entraron "desgreñadas" o desordenadas, delgadas o "enflaquecidas", desnutridas, entre otros calificativos que, según las concepciones de la imagen femenina de la locura, "en el semblante y en la gesticulación del cuerpo podemos leer una subversión de los códigos sociales y culturales que llevó al alienismo y la psiquiatría a patologizar y clasificar visualmente cualquier estado contrario al ideal normativo" (Montilla, 2016, p. 80). En efecto, la fisonomía de la bruja en la edad media, fue transpuesta en la histérica del siglo XIX, que Charcot y Freud remitieron desde los expedientes de la Inquisición, régimen que también refieren Szasz (2006, 87-88) y Ackerknecht (1993, p. 18 ) en sus historias de la psiquiatría.

Según Szasz, los psiquiatras franceses Pinel, Esquirol y Charcot, fueron "los responsables directos - dentro del campo médico- de la clasificación de las brujas como pacientes mentales". Pinel, en su Tratado sobre la locura (1801), afirmó que "en resumen: los endemoniados de todo tipo deben ser clasificados entre los maniacos o entre los melancólicos. Por su parte, Esquirol fue quien más influyó en que el acusado de brujo se enviara al asilo psiquiátrico en lugar de ir a la cárcel por impostor (Szasz, 2006, pp. 85-86).

Esta imagen pervivió hasta la primera mitad del siglo XX; no en vano, la presencia de la bruja en el contenido delirante estuvo asociada con la envidia (o ser miradas con malos ojos), intriga y calumnia por parte de vecinas, coasiladas y otras mujeres, así como ser tentadas por el diablo.

De hecho, el retrato pintado por Gèricault llamado "La monomanía de la envidia" (1820), por encargo de Georget - discípulo de Esquirol en la Salpêtrièrealude a que esta conducta era considerada particularmente un problema femenino, en tanto, "el afán de ascenso social de las féminas será visto como un mal o vicio moral que infringía la ética burguesa, una patología. La envidia será entendida no en su acepción positiva (deseo de emulación, afán de ser como alguien) sino enfermiza (perversidad nacida de la soberbia y pecado capital). Según cómo y hasta dónde se emule será un vicio y, en tanto que vicio, locura (Montilla, 2016, p. 82).

Según la tesis de Pedro Anzola se consideró a la envidia como una forma de locura moral que la padecían más las mujeres que los hombres (Anzola, 1904, p. 12). En Colombia, la visión femenina de la época estuvo influida por el degeneracionismo, que afirmaba el prejuicio de que "el misticismo domina a la mujer, sobre todo, que depositaria inmaculada de la moralidad de la raza, se desvía hacia el idealismo asceta..." (López de Mesa, 1915, pp. 115-117), puesto que estas actitudes menguaban la capacidad de defensa del hogar que debía asumir frente a la decadencia física, moral y mental, sobre todo el riesgo del alcoholismo declarado como "enfermedad social".

Asimismo, el ideal de la mujer estuvo consagrado por el dogma de la Inmaculada Concepción (invocada en el contenido delirante de pacientes) que, desde el siglo XVI estuvo presente como icono de hospitales coloniales y en la imaginería religiosa de la época en Colombia. Desde entonces, se instauró la devoción mariana, culto que tuvo enorme difusión y arraigo, que influyó en la imagen de la madre en valores de pureza, abnegación y sacrificio (Rodríguez, 2004, p. 273). 


\section{5. "SANTA LOCURA" O "ABNEGACIÓN INCOMPRENDIDA" EN LA MADRE LAURA MONTOYA}

La Beata Laura Montoya Upegui (1874-1949), primera santa colombiana canonizada en 2013 , célebre escritora de prosa poética y mística, en su autobiografía relató cómo sufrió una enfermedad neuropsiquiátrica a la edad de siete años, por la que fue declarada "incurable" con el diagnóstico del "Baile de San Vito" o Corea (que viene del latín chorea, baile), asociado a la fiebre reumática causada por la infección por estreptococos, esta se caracteriza por movimientos involuntarios anormales, la cual si bien era poco usual, algunos casos eran tratados en manicomios, como el Departamental de Antioquia.

Una reconstrucción de su historia clínica evidencia que a la edad de 75 años sufrió de linfangitis crónica, coloquialmente conocida como "envenenamiento de la sangre", asociada a la obesidad grave que desarrolló desde la adultez temprana, y que ocasionaron su muerte en 1949 por una insuficiencia cardiaca congestiva. Sin embargo, el análisis científico realizado no incluyó el diagnóstico temprano de la enfermedad de Corea, ni en posteriores desordenes nerviosos y estados de delirio que pudieron ocasionar las infecciones y las fiebres altas, entre otras causas (Parra, 2018, pp. 170-172).

Un segundo momento de experiencia con la enfermedad, lo vivió a la edad de 16 años en 1890, cuando por una urgencia tuvo que reemplazar varios meses a la directora de la Casa de Locos de Medellín, María Jesús Upegui Moreno quien fuera su tía y en actual proceso de beatificación. Fue entonces cuando habitó la Casa a cargo de un centenar de hombres y mujeres durante varios meses, labor que desempeñó con una entrega tal que ella misma decía haberse convertido en una loca por el cuidado de los locos, "parecía una vieja directora de manicomios..." (Montoya, 1971 / 2008, p. 112).

\subsection{TOCADA POR EL RAYO DE DIOS, EMBRIAGUEZ ESPIRITUAL Y DELIRIO DE AMOR DIVINO}

En su testimonio de vida, en un apartado del capítulo II, "Primera gracia extraordinaria" relata la escena más característica de su experiencia extática, en la que sintió en su cuerpo una energía como si fuera eléctrica, que la atribuyó a la posesión de la presencia divina:
Aquel rayo fue un conocimiento de Dios y de sus grandezas, tan hondo, tan magnífico, tan amoroso... Por fin terminé llorando y gritando recio, recio, como si para respirar necesitara de ello. Por fortuna estaba a distancia de ser oída de los de la casa. Lloré mucho rato de alegría, de opresión amorosa, y grité... Volvía los ojos al cielo y gritaba, llamándolo como una loca. Lloraba porque no lo veía y gritaba más. Siempre el amor se convierte en dolor. Este casi me mata (Montoya, 1971 / 2008, p. 61).

En el capítulo IX, "Supremo dolor del pecado", sobre su sensación de contrición y delirio de amor divino, expresó: "Mi interior todo era clavos, sangre, cruz, pretorio, calvario, espinas... Las lágrimas eran la savia con que salpicaba mi alma; estas cosas y un amor parecido a locura, acompañaban mi oración" (Montoya, 1971 / 2008, p. 154).

En el capítulo X, “Estudiar a Dios es el cielo", relató su experiencia de erotismo, éxtasis y delirio místicos: "Unos gozos de los atributos de Dios, me sacaban de casillas, como suele decirse y me ponían loca... que llamo locura ¿será lo que llaman embriaguez espiritual? Sobre todo, con la vida divina tuve un día una terrible que me hizo lanzar gritos locos. Por fortuna, estaba sola en un huerto y nadie vio ni oyó aquel escándalo (Montoya, 1971/2008, p. 168). Esta escena la describe en otros lugares de su testimonio como el momento en que fue "tocada por el rayo de Dios".

En el capítulo XIX, "Cedí las gracias", reitera la experiencia de incomprensión de sus acciones y declara la que va a ser su consigna combativa frente a los señalamientos de intransigencia y delirio: "Pero, como la locura es la característica de mi alma, estampo estas cosas aquí, con temor de escandalizar a quienes no alcancen a conocer muy bien las apreturas del amor encerrado en un miserable e impotente cuerpo de barro" (Montoya, 1971 / 2008, p. 179).

En el capítulo XXIX, "Cómo se mitigaban mis dolores", recurre a la sensación extática anteriormente descrita para afirmar su convicción de una legítima locura validada en el discurso de la mística cristiana:

Perdóneme padre de mi alma este arranque del cual sin embargo no puedo arrepentirme, porque él no expresa aun lo que siento, aunque sí me parece que estoy loca... En estos días le pregunté al señor Builes si las almas se enloquecían y me contestó que sí... Pues desde entonces me enloquecí. ¿No es locura llamar a Dios como entonces lo llamaba? ¡Yo creo que gente más juiciosa se 
contiene y además no se acorrala tanto en esas agonías! (Montoya, 1971 / 2008, p. 455).

\subsection{EVANGELIZAR A LOS "INDIOS INFIELES", UNA "SANTA LOCURA"}

En 1914, la misión que emprendió la fundadora de la comunidad de las "Lauritas" en Colombia, consistió en la travesía de un grupo de mujeres jóvenes desde Medellín a la región selvática de Dabeiba en el departamento de Antioquia, con el propósito de intervenir con la catequesis a la comunidad indígena $\mathrm{Ca}-$ tíos, asentamiento donde se instalaron durante 11 años hasta 1925. Para la época, esta fue una arriesgada aventura que les valió la acusación de encontrarse en una verdadera locura. Así lo expresa en el capítulo XVIII, "Sólo la gloria de Dios":

A muchos les parecía loca. Por eso me presenté una vez al padre Muñoz y le dije: - Padre, a los locos les siguen la idea o los llevan al manicomio. Si a mí me llevaran a aquel lugar, me harían mucha caridad porque allí al menos podré decirle a Dios: estas murallas me impidieron y quedaré tranquila convencida de mi locura. El padre se rió y me dijo: -Es que no está tan loca que merezca el manicomio; pero si sigue, seguramente, habrá que llevarla (Montoya, 1971 / 2008, p. 277)

En el capítulo XX, "Quise servir hasta para rueda de carro", narra todas las actividades para reunir fondos para el viaje, muchas de ellas controversiales, como la del establecimiento de la adoración nocturna para las mujeres, según ella, "una de las tantas locuras que el deseo de esa gracia, que veía tan lejos, me hizo inventar" (Montoya, 1971 / 2008, p. 314).

En el capítulo XXI, "Dios dirige las cosas cuando son suyas", cuenta que un sacerdote en el confesionario la increpó: "Le prohíbo seriamente continuar en la empresa de los indios y aún pensar en ellos. Eso no es factible ni es para su sexo, ni tiene trazas sino de una gran locura" (Montoya, 1971 / 2008, p. 314). En el capítulo XXXII, "Abnegación incomprendida", un médico de confianza de la familia de la madre Laura las abordó en el camino y le dijo:

Mira, yo quiero ver a tus hijas y saber si están locas... - ¡Es que yo sé quiénes son ellas y lo que en Medellín dejaron y sólo estando locas rematadas, pueden haber venido a buscar estos negros tan asquerosos, sometiéndose a estas cosas! Eso de consumir una juventud en una quijotada es cosa muy seria y, además, al principio creí que era espíritu de novelería y que pronto regresarían; pero la cosa va larga y todos nos hemos quedado esperándolas. Dime, ¿qué las detiene o qué esperan? ¡Creo que sólo por un arranque de locura insigne pueden consumirse en esta clase de trabajos! - No te equivocas, le dije, sí, están locas, pero de una locura desconocida para ti y para cuantos ignoran lo que es amor de Dios. Ya de esta locura no se curarán; pero a ellas las hace felices. No te explico, porque no me entenderías jamás (Montoya, 1971 / 2008, pp. 516-517).

Contra todos sus opositores y obstáculos, las Lauritas lograron su osada misión en tiempos de hegemonía conservadora y en los que el rol social de la mujer era considerado pasivo e inequitativo, razón por la que desobedecer los impedimentos interpuestos era un síntoma de rebeldía, conducta que para la época era patologizada en el género femenino en términos de manía, histeria y delirio.

De hecho, en 1906 se pronunció en su "Carta abierta al doctor Alfonso Castro autor de Hija espiritual", por tratarse de una novela en la que el médico recrea a su propia hermana como a una "maestra monstruosa e impura; una falsa beata execrable y disociadora que, por envidia de solterona y por manía conventual, sugestiona y trastorna... una loca lastimosa y risible que arrulla una astilla de leña como al hijo de sus entrañas" (Montoya, 1906, pp. 4-5). Se trató de una larga defensa, según ella, por la "disección psicológica" que el autor hizo de las beatas, en tiempos en los que sufrieron del escarnio público, razón por la que Laura recibió la orden de la Arquidiócesis de Medellín para detener todo el alboroto por sus acciones no ortodoxas para la época (Rodríguez, 2018, pp. 2028). La lucidez y fuerza de los argumentos en su carta contradecían las habladurías de su sinrazón y transgresiones al orden.

\section{DISCUSIÓN}

Según las historias clínicas de los primeros asilos psiquiátricos de Colombia (1903-1960), la exaltación de ideas religiosas confluye en un delirio polimorfo, relacionadas con: persecución y sentimiento de rechazo; megalomanía y sentimiento de ambición; hipocondría o depreciación del cuerpo; erotomanía o exaltación sexual; celotipia o depreciación erótica; condenación y autoinculpación.

De esta forma de delirio emergieron seis categorías: la moderación de la devoción y la blasfemia como signo de perturbación; el manicomio, un infierno dantesco; sentirse en el cielo, en el otro mundo; asedio 
del diablo y otros espíritus; asedio de brujas; ver, oír, personificar a la Virgen y otros santos; exaltación de ideas místico-religiosas en el delirio polimorfo.

Los síntomas más recurrentes fueron las alucinaciones visuales y auditivas, logorrea o locuacidad, insomnio, negativismo alimentario acompañado de miedo a ser envenenado, excitación psicomotora o hiperquinética, accesos de furia controlados con camisa de fuerza y otros medios de contención, intento de suicidio y tendencia a la fuga de la casa, por lo tanto se consideraba de peligrosidad para sí mismos y para los demás, conductas que concuerdan con los casos expuestos por Pinel en La Salpêtrière, a principios del siglo XIX.

Entre las psicopatologías más recurrentes en las que se manifestaron los delirios se encuentra el grupo de las manías, principalmente la intermitente, psicosis maniaco-depresiva y melancolía; seguido por la esquizofrenia paranoide, y también fue frecuente en la psicosis puerperal o posparto; asimismo, se presentó en la parálisis general progresiva (neurosífilis) y en el síndrome cerebral agudo o estado de confusión agudo, nosografía moderna dada al delirio.

Los cuadros clínicos observados son similares a los referidos por Delacroix (1908) y Rosen (1974) sobre el éxtasis y la locura por posesión, así como en GarcíaAlbea (2012, pp. 91-112) en el caso de Teresa de Ávila, y en Pires (2016, pp. 93-118) sobre la histeria religiosa en Portugal. Por otra parte, se encuentra el estudio de Sacks (2013) sobre las alucinaciones, en particular sobre las migrañas visuales, en las que se perciben auras, sonidos, voces, tactos, etc., y puede llegar a sentirse levitaciones que, en lo cotidiano se asumen como experiencias místicas o "llamadas de Dios"; como se vio en los expedientes analizados en Colombia, era frecuente el dolor de cabeza intenso, sensación de ensanchamiento y estiramiento de la misma durante los accesos delirantes.

Según Sacks (2013), se han expuesto pruebas sobre este fenómeno en algunos estudios, entre ellos el de Kevin Nelson The spiritual doorway in the brain: a neorologist's search for the God experience (La puerta espiritual en el cerebro: la búsqueda de un neurólogo por la experiencia de Dios, 2011). También refiere la novela Lying awake (Acostada despierta, 2000), de Mark Salzman, cuya protagonista es una joven monja carmelita que sufre ataques extáticos en los que entra en comunión con Dios. Resulta que los ataques están provocados por un tumor en su lóbulo temporal, y hay que extirparlo antes de que sea grande y la mate. Pregunta el autor, pero ¿si se lo extirpan, le arrancarán también su portal al cielo, impidiendo que vuelva a estar en comunión con Dios?

Los "ataques extáticos" y sus alucinaciones están relacionados con algunos casos de epilepsia del lóbulo temporal, similar a lo referido por Sacks (2013), no en vano fue llamada "enfermedad sagrada" desde los griegos, quienes le atribuían su origen a una posesión o inspiración divina, hasta que Hipócrates se opuso a esta creencia, al definirla como una enfermedad natural que, por ignorancia y superstición, tuvo esas atribuciones por parte de brujos y charlatanes. Asimismo, esta reacción convulsiva estuvo rodeada de interpretaciones demonológicas en la Edad Media, con las consabidas consecuencias de la Inquisición. Así decía Hipócrates en su texto titulado Sobre la enfermedad sagrada:

...si se ha de considerar divina por sus extraordinarios rasgos, serán muchas las enfermedades sagradas y no una sola [...] Por un lado, las fiebres cotidianas, tercianas y cuartanas, cuyo carácter no se considera extraordinario, no me parecen en modo alguno menos sagradas y de origen menos divino que esta enfermedad; por otro lado, veo que los hombres están fuera de sí y deliran sin ninguna causa evidente y que realizan muchos actos intempestivos, y sé que muchos hombres gimen y gritan en sueños, que otros se ahogan y que otros saltan del lecho y huyen de su casa y están delirando hasta que despiertan, y que luego están sanos y cuerdos como antes, aunque pálidos y débiles; y esto ocurre no una sola vez, sino muchas" (1970, p. 87).

Más allá de los muros de las instituciones psiquiátricas, es conocida la historia médica de santas epilépticas como Juana de Arco (o "la loca"), Rosa de Lima, vulgarmente llamada "Santa loca de Lima", por sus ataques y delirios, así como los controvertidos diagnósticos de histeria que pesaron sobre Teresa de Ávila, llamada por Janet "santa patrona de las histéricas, cuando experimentaba sentimientos intensos de "bienestar", "placer" (no sexual), "plenitud", "paz", "belleza", entre otras calificaciones, en las que se revela una capacidad de autoobservación y de descripción para narrar lo "indescriptible" y relatar con precisión estos "inefables" sentimientos y estados de conciencia (García-Albea, 2012, pp. 91-112), virtudes literarias de las que también gozó Laura Montoya en su obra escrita.

La misión de la santa colombiana constituyó una resistencia a la patologización del delirio místico-religioso, una actitud inconfesa antipsiquiátrica. En su 
testimonio revela la experiencia de institucionalización del delirio como consigna y actitud exigida en su misión evangelizadora, causa por la que luchó en contra de las acusaciones de que estaba loca y, por el contrario, apropió una retórica del delirio con la que caracterizó sus acciones de "santa locura" por la obra de Dios.

No obstante, frente a esta patologización de la devoción se plantea una crítica a la "hiperreligiosidad" atribuida a la epilepsia extática, que tradicionalmente obedece a interpretaciones filosóficas, ultramorales, sentimientos de culpa, entre otros lugares comunes; de este modo, se plantea una oposición a que se acepte la religiosidad como una categoría clínica, puesto que un individuo hipo o hiperreligioso correría el riesgo de ser considerado un enfermo (García-Albea, 2012, pp. 91-112).

La religión en el contexto colombiano es una forma simbólica fundamental de la cultura, por lo tanto, son predecibles sus expresiones en el contenido delirante de los pacientes, de ahí que, no toda alusión teológica y demonológica sea suficiente para declarar el delirio místico-religioso propiamente dicho (Miranda, 2004, pp. 172-181).

Diversos estudios epidemiológicos evidencian que las creencias religiosas también contienen atributos asociados a la voluntad de recuperación de la salud mental, a saber, conciencia de Dios, aceptación de la

\section{BIBLIOGRAFÍA}

Ackerknecht, Erwin (1993), Breve historia de la psiquiatría, Seminari d'Estudis sobre la Ciència, Buenos Aires, Editora Universitaria.

Alvarado, Francisco (1904), Delirio de persecución, Tesis para el doctorado en Medicina y Cirugía, Universidad Nacional de Colombia, Colección de Libros raros y manuscritos, BLAA, Bogotá.

Anzola, Pedro (1904), De la locura moral, Tesis para el doctorado en Medicina y Cirugía, Universidad Nacional de Colombia, Bogotá, Imprenta Hernando Santos.

Berman, David (2006), "Religion and madness", Journal of Religion and Health, 45(3), 359-370.

Buendía, Nicolás (1893), Las monomanías impulsivas. Estudio clínico y médico-legal, Tesis de doctorado en Medicina y Cirugía, Universidad Nacional de Colombia, Bogotá, Imprenta La Luz. gracia y el amor de Dios, el arrepentimiento y la responsabilidad social, fe y confianza, participación en la religión organizada, compañerismo, ética, tolerancia y apertura a las experiencias de los demás (Moreira-Almeida, Lotufo-Neto, Koenig, 2006, pp. 242250). La correlación entre religión/espiritualidad y salud pública ( $\mathrm{R}$ / S-salud) ha cobrado interés en los últimos 20 años, en los que se publicó un centenar de revisiones sistemáticas y una treintena de metanálisis sobre R / S-salud. En efecto, en el campo de la salud pública tienen relevancia las implicaciones que cruzan entre la medicina, la psicología y la enfermería, disciplinas donde los factores R / S tienen mayor interés en la cuestión cultural de la salud colectiva (Oman, 2018).

Frente a la pregunta ¿cómo podemos distinguir la creencia genuina en Dios de la creencia loca en Dios, o, más generalmente, la religiosidad sana de la locura religiosa? Se asume que no hay una manera objetiva y ética de hacer la distinción y que una de las posturas más aceptables puede ser la neurótica, en tanto, la locura y la cordura, la religión y el ateísmo, pueden ser vistos como dos extremos entre los medios neuróticos (Berman, 2006, pp. 359-370). Según lo comentado sobre la interpretación psicoanalítica, se atribuye una analogía entre el ritual obsesivo y el ritual religioso, en tanto ambos se producen por sentimientos de culpa y deseos reprimidos, cuyos mecanismos de defensa recurren a lo ritual.
Delacroix, Henri (1908), Etudes d'histoire et de psychologie du mysticisme: les grands mystiques chrétiens, Paris, Félix Alcan, Éditeur, Bibliothèque nationale de France, gallica.bnf.fr

De Jaucourt, Louis (1751), "Scrupule", en: Diderot, Denis y D’Alembert, Jean, (1751), L'Encyclopédie, http://xn--encyclopdie-ibb.eu/index.php/877250295-SCRUPULE

De los Ríos, Alfredo (1981), "Un siglo de psiquiatría en Antioquia”, Boletín Comité de Historia de la Medicina, Medellín, 3(1).

Duguet, Jacques (1718), Traité des scrupules, de leurs causes, de leurs espèces, de leurs suites dangereuses, de leurs remèdes généraux \& particuliers, Paris, Jacques Estienne.

García-Albea, Esteban, García-Albea, Julia (2012), “La epilepsia de la carmelita Teresa de Jesús", en: Sáenz, M., Valiente C. y Fuentenebro, F. (Eds.), Los umbrales de la locura: una aproximación fenomenológica, histórica y cultural, Madrid, Editorial Complutense. 
Genicot, Eduardus (1931), Institutiones theologiae moralis, Lovaina, en: Compagnoni, F., Piana, G., Privitera S. (Dirs.) (1992), Nuevo diccionario de teología moral, Madrid, Paulinas.

Grimes, M. l'Abbe (1854), Traité des scrupules: instructions pour éclairer, diriger, consoler et guérir les personnes scrupuleuses, Paris, Pierre Tequi, https://archive.org/details/ MN5104ucmf_2/page/n4

Guislain, Joseph (1852), Leçons orales sur les phrénopathies. Gand, L. Hebbelynck, https://gallica.bnf.fr/ark:/12148/bpt6k76639j.textelmage

Goffman, Erving (2009), Internados, Ensayos sobre la situación social de los enfermos mentales, Buenos Aires, Amorrortu.

Gutiérrez, Jairo (2019). Locura y sociedad. Alienismo tardío, psicopatología e higiene mental en la modernidad, 18701968, Envigado, IUE.

Hipócrates (1970), Sobre la enfermedad sagrada (Trad. José Alsina), Boletín del Instituto de Estudios Helénicos, 4(1), p. 87.

Huertas, Rafael (2014), "Las obsesiones antes de Freud: historia y clínica", História, Ciências, Saúde Manguinhos, 21(4), 1397-1415.

Janet, Pierre (1903), Les obsessions et la psychasthénie, Paris, Alcan, https://archive.org/details/lesobsessionsetl02jane/ page/n6

Jiménez, Miguel (1920), Nuestras razas decaen. Algunos signos de degeneración colectiva en Colombia y en los países similares. Memoria presentada al Tercer Congreso Médico Colombiano reunido en Cartagena en enero de 1918, Bogotá, Imprenta y Litografía de Juan Casis.

López De Mesa, Luis (1915), “El problema del alcoholismo y su posible solución", Repertorio de Medicina y Cirugía, Bogotá, 7(3), 115-117.

Miranda, Carlos (2004), "Vigencia del delirio místico en la semiología contemporánea", Revista Colombiana de Psiquiatría, 33(2), 172-181.

Montilla, Julia (2016), Enajenadas. Ilustraciones médicas de la locura femenina en el siglo XIX, Madrid, Brumaria.

Montoya, Laura (1971/2008), Autobiografía o historias de las misericordias de Dios en un alma, Medellín, Bedout / Cargraphics.

Montoya, Laura (1906), Carta abierta al doctor Alfonso Castro autor de Hija espiritual, Medellín, El Comercio.

Moreira-Almeida, Alexander; Lotufo-Neto, Francisco; Koenig, Harold (2006), Religiousness and mental health: a review. Rev Bras Psiquiatr., 28(3), 242-50.
Oman, Doug (Ed.) (2018), Why religion and spirituality matter for public health. Evidence, implications, and resources, Switzerland, Springer.

Parra, Paola (2018), "La muerte de una santa: bosquejo de una historia clínica” (152-175), en: Osorio, María. (ed.) (2018), Discursos y prácticas en la vida y obra de Santa Laura Montoya, Berlin, Peter Lang.

Pinel, Philippe (1809/2012), Tratado médico filosófico sobre la alienación mental, Paris, J. Ant. Brosson, Libraire, Reimp. Stagnaro, Juan (Dir. Comp.) (2012), El nacimiento de la psiquiatría: Haslam, Reil, Esquirol, Pinel, Buenos Aires, Polemos.

Pires, Tiago (2016), "Intimaçoes do êxtase feminino: dois momentos da cultura da histeria em Portugal", en: Marmitt, Yonissa (Ed.) (2016), Narrativas sobre loucuras, sofrimentos e traumas, Curitiba, Máquina de escrever.

Putnam, Carlos (1913), "Informe presentado a la Academia Nacional de Medicina", Revista Médica de Bogotá, 31, pp. 248-259.

Rodríguez, Flor (2018), "Laura Montoya Upegui: conductas, creencias y emociones de 1904 a 1906..." (19-44), en: Osorio, María (ed.) (2018), Discursos y prácticas en la vida y obra de Santa Laura Montoya, Berlin, Peter Lang.

Rodríguez, Pablo (2004), La familia en Iberoamérica 1550-1980, Bogotá, Universidad Externado de Colombia.

Rosen, George (1974), Locura y sociedad, sociología histórica de la enfermedad mental, Madrid, Alianza.

Rosselli, Humberto (1968), Historia de la Psiquiatría en Colombia, T. 1 y 2, Bogotá, Horizonte.

Sacks, Oliver (2013), Alucinaciones, Buenos Aires, Anagrama.

Szasz, Thomas (2006), La fabricación de la locura. Estudio comparativo de la Inquisición y el movimiento de defensa de la salud mental, Barcelona, Kairós.

Tanquery, Adolphe (1930), Précis de théologie ascétique et mystique, París, Desclée \& Cía., http://patrimoinechretien. com/pc-content/uploads/2016/04/Pr\%C3\%A9cis-deth\%C3\%A9ologie-As\%C3\%A9tique-et-Mystique.pdf

Taylor, Jeremy (1660), Ductor dubitantium: or, The rule of conscience in all her general measures, London, Norton \& Royston.

Uribe, Lázaro (1923), "Principales factores etiológicos de la locura en los departamentos de Antioquia y Caldas", Revista clínica, Sociedad clínica del Hospital de Medellín, 3(25-28), 188-198.

Varieté (1907), "L'hystérie des mystics", Journal des Practiciens, s.f., 305, traducción en: Anales de la Academia de Medicina de Medellín, 1907, 14, pp. 177-179.

Vasco, Eduardo (1948), Temas de higiene mental, educación y eugenesia, Medellín, Bedout. 